\title{
Laser Guiding for GeV Laser-Plasma Accelerators
}

\author{
By Wim Leemans ${ }^{\dagger}$, Eric Esarey ${ }^{\dagger}$, Cameron Geddes, Carl Schroeder, \\ AND CSABA TÓTH \\ LOASIS Program, Accelerator and Fusion Research Division, Lawrence Berkeley \\ National Laboratory, Berkeley, CA 94720, USA
}

\begin{abstract}
Guiding of relativistically intense laser beams in preformed plasma channels is discussed for development of GeV-class laser accelerators. Experiments using a channel guided laser wakefield accelerator (LWFA) at LBNL have demonstrated that near mono-energetic $100 \mathrm{MeV}$-class electron beams can be produced with a 10 TW laser system. Analysis, aided by particle-in-cell simulations, as well as experiments with various plasma lengths and densities, indicate that tailoring the length of the accelerator, together with loading of the accelerating structure with beam, is the key to production of mono-energetic electron beams. Increasing the energy towards a $\mathrm{GeV}$ and beyond will require reducing the plasma density and design criteria are discussed for an optimized accelerator module. The current progress and future directions are summarized through comparison with conventional accelerators, highlighting the unique short term prospects for intense radiation sources based on laser-driven plasma accelerators.
\end{abstract}

Keywords: Laser, Plasma, Accelerator, Wakefield, Guiding

\section{Introduction}

Laser-driven plasma accelerators, in which large amplitude plasma waves are excited by an intense laser beam, were proposed in 1979 by Tajima \& Dawson (1979). Experiments conducted during more than two decades that followed this seminal paper, demonstrated that by focusing intense laser pulses onto a neutral gas, relativistic electron beams can be produced (Modena et al. 1995; Umstadter et al. 1996a; Esarey et al. 1996). The accelerated electron energy spectrum was characterized by an exponential or Boltzmann-like distribution, with the majority of electrons at modest energies (a couple of $\mathrm{MeV}$ ). The total accelerated charge was large (up to several $\mathrm{nC}$ ), but the number of electrons at high energy (tens of $\mathrm{MeV}$ ) was an exponentially small fraction of the total charge. Over the years, the quality of these bunches improved, and beams were produced with "smaller" lasers, capable of operating at higher repetition rate (Malka et al. 2002). Previous generation experiments utilized large, single shot, high power $\mathrm{CO}_{2}$ (Clayton et al. 1993) or Nd:glass lasers (Modena et al. 1995). The "smaller" lasers rely on $\mathrm{Ti}^{2} \mathrm{Al}_{2} \mathrm{O}_{3}$ for amplifying medium and on chirped pulse amplification (Strickland and Mourou $1985)$ and are capable of producing 30 fs duration pulses containing several Joules

$\dagger$ Also at the Department of Physics, University of Nevada, Reno 
of energy at a repetition rate of $10 \mathrm{~Hz}$. Laser pulse shape effects were studied (Leemans et al. 2002; Schroeder et al. 2003) (enabled by the CPA architecture of the laser), and applications were explored such as radio-isotope production (Santala et al. 2001; Leemans et al. 2001; Ledingham et al. 2003), THz radiation generation (Leemans et al. 2003; Schroeder et al. 2004), and x-ray generation (Leemans et al. 2000; Catravas et al. 2001; Esarey et al. 2002; Rousse et al. 2004). Although steady progress had been made, the 100 percent energy spread remained a major limitation.

Recent simulations of laser-plasma interaction in an ultra-relativistic regime indicated however that the generation of quasi-monochromatic bunches (Pukhov \& Meyer-ter-Vehn 2002) was possible. The bubble or blow-out regime is well known in plasma wakefield accelerators, when using electron beams denser than the surrounding plasma (Rosenzweig et al. 1991). In the case of lasers, it occurs when the ponderomotive potential of the laser is large enough to blow out all plasma electrons. However, the simulations (Pukhov \& Meyer-ter-Vehn 2002) were done for incident laser intensities that were not (yet) achievable experimentally. As it turns out, through an interplay between the laser pulse and plasma, the self-modulation instability leads to laser pulse steepening, which allows this regime to be accessed experimentally.

In 2004 a major new milestone was reported in the journal Nature, with the production and measurement of high quality electron bunches. Three different groups [located at the Rutherford Appleton Laboratory-RAL (UK), Lawrence Berkeley National Laboratory-LBNL (USA) and the Laboratoire d'Optique Appliquée-LOA (France)] announced measurement of electron bunches with narrow energy spread containing a significant amount of charge in a beam with a small divergence. In the case of the LBNL experiments, this was accompanied by the achievement of another major milestone: the guiding of relativistically intense $\left(>10^{18} \mathrm{~W} / \mathrm{cm}^{2}\right)$ laser pulses within preformed plasma channels and the self-trapping and acceleration of electrons within these channels. As will be discussed, guiding of high intensity laser pulses in plasma channels is necessary in order to extend the acceleration length and the energy gain up to the multi-GeV range with reasonable size laser systems that can operate at high repetition rates.

To obtain the mono-energetic bunches, the RAL and LOA groups used relatively large laser spot sizes. This effectively increases the diffraction (or Rayleigh range, $Z_{R}$ ) of the laser beam permitting propagation over distances on the order of the gas jet length. The RAL-AlphaX collaboration used a $16 \mathrm{TW}, 40$ fs laser pulse focused (25 $\mu \mathrm{m}$ spot size, $\left.1 \times 10^{18} \mathrm{~W} / \mathrm{cm}^{2}\right)$ on a plume of a gas jet with a plasma density of $2 \times 10^{18} \mathrm{~cm}^{-3}$. A narrow energy spread bunch was observed at $78 \pm 2 \mathrm{MeV}$ with $20 \mathrm{pC}$ of charge (Mangles et al. 2004). The LOA experiments used a $30 \mathrm{TW}, 33 \mathrm{fs}$ laser pulse focused $\left(18 \mu \mathrm{m}\right.$ spot size, $\left.3 \times 10^{18} \mathrm{~W} / \mathrm{cm}^{2}\right)$ on a plume of a gas jet with a plasma density of $6 \times 10^{18} \mathrm{~cm}^{-3}$. A narrow energy spread bunch was observed at $170 \pm 15 \mathrm{MeV}$ with $500 \mathrm{pC}$ of charge (Faure et al. 2004).

The LBNL experiments used a $9 \mathrm{TW}, 55$ fs laser pulse focused to a relatively tight spot size $\left(8.5 \mu \mathrm{m}\right.$ FWHM). To mitigate the short $Z_{R}$ of the beam, a preformed plasma channel was used to guide the laser beam through the gas jet. Channel guiding, including the method used at LBNL, will be described next. This is followed by a discussion on scaling laws that indicate the importance of development of long plasma channels for $\mathrm{GeV}$-class accelerators. The manuscript will conclude with a 
discussion on the state-of-the-art performance of LWFAs and future developments that are needed the enable the use of laser driven accelerators for various applications.

\section{Channel guiding: scaling laws and experiments}

The importance of the accelerator length, and how to extend it, has been discussed theoretically for many years. As an example, during the 1995 Kardamyli Workshop on second generation plasma-based accelerators, design studies were carried out in the laser guiding and acceleration working group, for a $1 \mathrm{GeV}$ LWFA (Leemans et al. 1996). The main conclusion of the study was that extending the propagation of a laser pulse beyond $Z_{R}$, up to the dephasing distance (Esarey et al. 1996), was an essential element of a future LWFA. Much higher net energy gains can be realized for the same amount of input laser power by extending the acceleration distance beyond $Z_{R}$. Guiding concepts relying on the use of preformed channels are being studied by several groups around the world (Volfbeyn et al. 1999; Geddes et al. 2004a; Alexeev et al. 2003; Downer et al. 2003; Tochitsky et al. 2004; Sprangle et al. 2001; Spence \& Hooker 2001; Zhidkov et al. 2004; Lopes et al. 2003; Chen et al. 2004; Cros et al. 2002). At LBNL, using the ignitor-heater concept (Volfbeyn et al. 1999), we have recently produced plasma channels that have guided, to our knowledge, the highest peak power and the highest intensity in a preformed channel (Geddes et al. 2004a). This result demonstrates the ability of plasma channels to guide laser beams, at intensities relevant for particle acceleration, over many $Z_{R}$. After a brief review of scaling laws in Section 2a, experimental results from the LOASIS group on laser guiding and electron acceleration will be summarized in sections $2 \mathrm{~b}$ and $2 \mathrm{c}$, respectively.

\section{(a) Scaling laws}

There are several mechanisms that can limit the effective accelerator length, and, therefore, the energy gain in a LWFA (Esarey et al. 1996). One of the most severe is diffraction of the laser pulse, but this can be overcome through the use of a plasma channel to guide the laser pulse (Sprangle et al. 1992; Durfee \& Milchberg 1993; Esarey et al. 1997; Geddes et al. 2004a). The two main limitations for a channelguided LWFA are then electron dephasing and pump depletion. Electron dephasing is a result of a highly relativistic electron outrunning the wakefield, which typically has a phase velocity $<c$. In the low intensity (or linear) limit, $a_{0}^{2} \ll 1$, the dephasing length is $L_{d e p h} \sim \lambda_{p}^{3} / \lambda_{0}^{2}$, assuming an axially uniform, wide plasma channel, where $\lambda_{p}$ is the plasma wavelength, $\lambda_{0}$ is the laser wavelength, and $a_{0}$ is the normalized peak vector potential of the laser field, which is related to the peak intensity of a linearly polarized laser pulse by $a_{0}=8.5 \times 10^{-10} \lambda_{0}[\mu \mathrm{m}]\left(I_{0}\left[\mathrm{~W} / \mathrm{cm}^{2}\right]\right)^{1 / 2}$. In the high intensity (or nonlinear) limit, $a_{0}^{2} \gg 1$, the dephasing length is $L_{\text {deph }} \sim\left(\lambda_{p}^{3} / \lambda_{0}^{2}\right) a_{0}$, where a constant of order unity has been neglected.

The laser pulse excites a wake as it propagates. Wake excitation leads to loss of laser pulse energy. The pump depletion length (the distance over which the pump loses a significant fraction of its energy) can be estimated by equating the laser pulse energy to the energy left behind in the wake (Horton \& Tajima 1986; Bulanov et al. 1992). In the linear limit $\left(a_{0}^{2} \ll 1\right)$, the pump depletion length is $L_{p d} \sim\left(\lambda_{p}^{3} / \lambda_{0}^{2}\right) a_{0}^{-2}$, 
whereas in the nonlinear limit $\left(a_{0}^{2} \gg 1\right), L_{p d} \sim\left(\lambda_{p}^{3} / \lambda_{0}^{2}\right) a_{0}$. Once pump depletion occurs, staging with a fresh pump pulse is necessary.

Scaling laws describing the energy gain in a standard LWFA (where the laser pulse length $L_{L}$ is on the order of the plasma wavelength $\lambda_{p}$ ) can be derived under idealized assumptions (Esarey et al. 1996; Leemans et al. 1996). Three different cases are considered. The first is for a uniform plasma wherein the acceleration length is limited by vacuum diffraction, i.e., $L_{a c c} \simeq \pi Z_{R}$, where $Z_{R}=\pi w_{0}^{2} / \lambda_{0}$ and $w_{0}$ is the laser spot size. The second is for a plasma channel wherein the acceleration length is limited by electron dephasing, and the third is a plasma channel wherein the acceleration length is limited by pump depletion. In all cases, the laser power is assumed to be less than the critical power for relativistic self-focusing (Esarey et al. 1996) and the effects of instabilities are neglected. Furthermore, the laser pulse and plasma are assumed to be wide and axially uniform, and the accelerating field is assumed to be that for an optimized flat-top pump pulse, i.e., $E_{\max } / E_{0}=$ $\left(a_{0}^{2} / 2\right)\left(1+a_{0}^{2} / 2\right)^{-1 / 2}$, where $E_{\max }$ is the maximum electric field amplitude of the wake behind the laser pulse and $e E_{0}=2 \pi m c^{2} / \lambda_{p}$. The ideal energy gain $\Delta W$ is given by multiplying $E_{\max }$ by the acceleration length.

The scaling laws for the ideal energy gain in a channel-guided LWFA, as obtained from analytic theory, can be summarized as follows (Esarey et al. 2004). If the acceleration distance is limited by diffraction, $L_{a c c} \simeq \pi Z_{R}<L_{d e p h}, L_{p d}$, the energy gain in practical units is

$$
\Delta W_{\text {diff }}[M e V] \simeq 740\left(\lambda / \lambda_{p}\right)\left(1+a_{0}^{2} / 2\right)^{-1 / 2} P[T W] .
$$

If the acceleration distance is limited by the dephasing length, $L_{a c c} \simeq L_{d e p h}$, the energy gain in practical units is

$$
\Delta W_{\text {deph }}[\mathrm{MeV}] \simeq \frac{630 I\left[\mathrm{~W} / \mathrm{cm}^{2}\right]}{n\left[\mathrm{~cm}^{-3}\right]} \times \begin{cases}1, & a_{0}^{2} \ll 1, \\ (2 / \pi) / N, & a_{0}^{2} \gg 1,\end{cases}
$$

where $N$ is the number of plasma periods that the bunch is located behind the laser pulse. If the acceleration distance is limited by the depletion length, $L_{a c c} \simeq L_{p d}$, the energy gain in practical units is

$$
\Delta W_{p d}[\mathrm{MeV}] \simeq \begin{cases}3.4 \times 10^{21} /\left(\lambda^{2}[\mu \mathrm{m}] n\left[\mathrm{~cm}^{-3}\right]\right), & a_{0}^{2} \ll 1, \\ 400 I\left[\mathrm{~W} / \mathrm{cm}^{2}\right] /\left(n\left[\mathrm{~cm}^{-3}\right]\right), & a_{0}^{2} \gg 1 .\end{cases}
$$

Note that the nonlinear (or mildly nonlinear regime) is advantageous, since $L_{\text {deph }} \sim L_{p d}$ which implies efficient use of the pump laser pulse energy in a single stage: a significant amount of the laser pulse energy is extracted when the electron beam is extracted. Furthermore, the energy gain and accelerating gradients are higher compared to the linear regime.

As an example, consider nonlinear $a_{0}^{2} \gg 1$. This is favourable since the dephasing length is approximately equal to the depletion length, which implies higher efficiency, in addition to larger accelerating fields and larger energy gains. Assuming a Ti:sapphire laser $(\lambda=0.8 \mu \mathrm{m})$ with $P=100 \mathrm{TW}$ (e.g. a 55 fs pulse containing $5.5 \mathrm{~J}$ ) focused to a spot size of $w_{0}=18 \mu \mathrm{m}$, one obtains $a_{0}=3$ and $I=1.9 \times 10^{19} \mathrm{~W} / \mathrm{cm}^{2}$. Focusing this beam on a plasma with $\lambda_{p}=33 \mu \mathrm{m}$ 
$\left(n_{0}=10^{18} \mathrm{~cm}^{-3}\right)$, such that $L_{L}=\lambda_{p} / 2$, gives a wakefield of $E_{z}=190 \mathrm{GeV} / \mathrm{m}$, an acceleration length of $L_{a c c}=3.8 \mathrm{~cm}$, and an energy gain of $\Delta W=7.2 \mathrm{GeV}$.

In the next section, recent experimental results will be discussed, that demonstrate that radially shaped plasmas can indeed guide intensities relevant to large amplitude wake excitation and particle acceleration.

\section{(b) Guiding relativistic intensities in plasma channels}

As discussed in the previous section, the ability to guide intense laser pulses over many $Z_{R}$ is an essential element of a high energy LWFA. Plasma channel guiding of short laser pulses was first demonstrated in hydrodynamically formed plasma channels produced by focusing a laser beam with an axicon lens (Durfee \& Milchberg 1993; Milchberg et al. 1996). In these pioneering experiments, high$\mathrm{Z}$ gases were used to facilitate the ionization process. High-Z gases, however, are susceptible to further ionization when used with ultra-high intensity lasers, and, therefore, a method was needed to allow the use of low-Z gases. By separating out the ionization and heating phase of the channel formation, channels were produced in hydrogen gas with the ignitor-heater method (Volfbeyn et al. 1999).

Recently, channeling at relativistic intensities was realized with the 10 TW multi-beam LOASIS laser (Geddes et al. 2004a). Preformed guiding channels were created using a variation of the ignitor-heater method (Volfbeyn et al. 1999). The schematic of the experiment is shown in Fig. 1. A plasma was formed in a $2.5 \mathrm{~mm}$ long supersonic $\mathrm{H}_{2}$ gas jet with an atomic density of $3-4 \times 10^{19} \mathrm{~cm}^{-3}$ by an ignitor pulse $(15 \mathrm{~mJ}, 60 \mathrm{fs})$ that is co-axial with the drive pulse, then heated by a heater pulse (150 mJ, $250 \mathrm{ps}$ ). Hydrodynamic expansion of the plasma formed a channel (Durfee \& Milchberg 1993; Milchberg et al. 1996) that guided a relativistically intense drive pulse that was focused at the entrance to the channel. The drive pulse (500 mJ, $55 \mathrm{fs}$ ) was focused with an off-axis parabola to a spot of $7-8.5 \mu \mathrm{m} \mathrm{FWHM}$ resulting in a laser intensity of $1.1 \times 10^{19} \mathrm{~W} / \mathrm{cm}^{2}$ ). Propagation of the laser was monitored with a side interferometer, mode imager CCD, and transmitted light spectrometer. Electrons accelerated by the plasma wake of the drive beam were analysed using an integrating current transformer (ICT), a phosphor screen, and a magnetic spectrometer. A $55^{\circ}$ dipole magnet permitted energy distribution measurements up to $92 \mathrm{MeV}$ with about $1 \%$ resolution and a range of $25 \%$ around a chosen central momentum. Higher energies (up to $150 \mathrm{MeV}$ ) were measured by operating the spectrometer at a $5^{\circ}$ angle, at the cost of reduced resolution $(10 \%$ level).

The ignitor-heater method provided several tuning knobs to tailor the channel properties. Varying the time delay between the heater and drive pulses, energy of the heater pulse and spatial overlap, channels can be created with different radial density profiles. Figure 2 shows an example of mode images of laser spots at $4 \mathrm{TW}$ $\left(7 \mu \mathrm{m}\right.$ input spot, $\left.7 \times 10^{18} \mathrm{~W} / \mathrm{cm}^{2}\right)$. With the channel on, the output spot (b) matches the input (a). The mode imager resolution is restricted by $\mathrm{f \#} \mathrm{constraints}$ in the target chamber, and measures a $12 \mu \mathrm{m}$ FWHM spot size for both input and output. The input beam size was independently measured using a higher resolution imaging system installed inside the vacuum chamber. This system however could only be used upstream of the gas jet. The pulse duration was measured using a single shot autocorrelator and was typically around $55 \mathrm{fs}$ in the experiments. The 


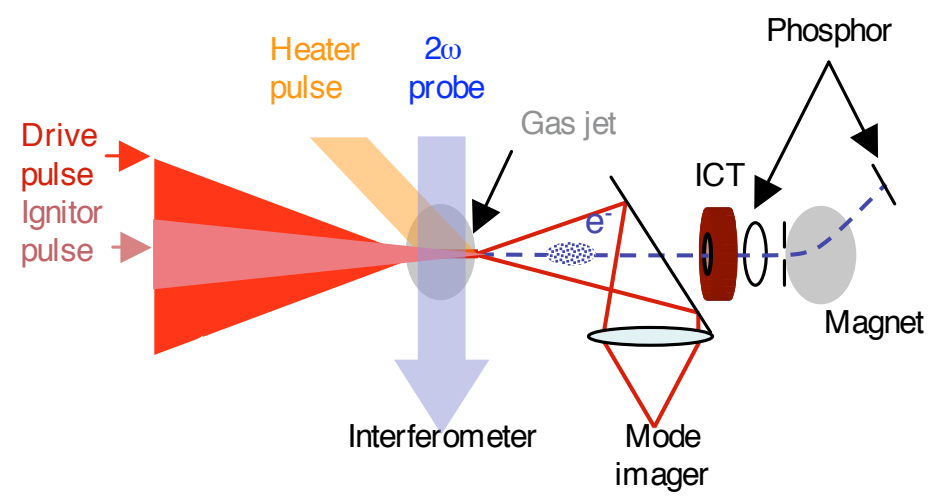

Figure 1. Schematic of the setup used in the channel guided laser wakefield experiments.

input energy per pulse was obtained by first using a power meter that measured the output energy of the main amplifier, then multiplying by the measured transmission efficiency from amplifier to gas jet. The total intensity of the beam collected by the mode imager was then calibrated to provide energy per pulse on each shot. A calibrated photodiode monitored the energy of every pulse out of the main amplifier to account for variation in shot-to-shot energy. Measurement of the spectrum of the laser beam indicated that minimal shifting (less than $100 \mathrm{~nm}$ ) of the laser spectrum occurred during the interaction with the channel which is a range over which the mode imaging system is spectrally insensitive. From these measurements, the guided intensity was between $10^{18}$ and $2.5 \times 10^{18} \mathrm{~W} / \mathrm{cm}^{2}$, with the lower limit set by the $12 \mu \mathrm{m}$ mode imager observation and the upper limit set by the input spot size. In the absence of any plasma, a large mode size consistent with vacuum diffraction is observed (c), and with the gas jet on but the channel off (d) diffraction is increased by ionization effects (Rankin et al. 1991; Leemans et al. 1992), showing that self guiding alone is insufficient to efficiently guide the beam. Energy transmission at $4 \mathrm{TW}$ was $35 \%$, a reduction of one third from the low power case at $0.5 \mathrm{TW}$, indicating that substantial power was deposited in plasma waves. The depletion observed, is consistent with particle-in-cell simulations, run with the experimental parameters. The simulations also indicate that a plasma wave averaging 200-300 GV/m is excited, in the last $0.5 \mathrm{~mm}$ of guide length. No electrons are self trapped at $4 \mathrm{TW}$, making this an attractive structure for controlled injection experiments (Esarey et al. 1997; Schroeder et al. 1999; Fubiani et al. 2004), as will be discussed below. When increasing the laser power up to the $10 \mathrm{TW}$ level, electron beams were produced with unprecedented properties, as discussed in section 2c. 




Figure 2. Mode images of laser propagation at $4 \mathrm{TW}$, or two times the critical power for self focusing. The guided output mode after $2.5 \mathrm{~mm}\left(10 Z_{R}\right)$ of propagation (B) is indistinguishable from the input mode (A). The effect of the channel can be seen by comparison to vacuum propagation over the same distance where the output mode is severely diffracted (C). Self guiding also does not maintain the spot over this distance due to instability, and the output mode with gas jet on but without the guide is displays enhanced diffraction (D). Note enlarged scale in (C),(D).

\section{(c) Production of low energy spread bunches}

Using the preformed channels with laser input powers at the 8-10 TW level, electron beams with narrow energy spread were observed (Geddes et al. 2004a). Using the $55^{\circ}$ fine resolution magnetic spectrometer, beams containing $2 \times 10^{9}$ electrons with two percent energy spread around $86 \mathrm{MeV}$ were observed with a divergence on the order of $3 \mathrm{mrad}$. Beams containing $10^{9}$ electrons at energies between 135-170 $\mathrm{MeV}$ were observed using the $5^{\circ}$ port of the magnetic spectrometer. An example of a narrow energy spread spectrum is shown in figure 3. The normalized geometric emittance, obtained from assuming that the bunch comes from a source approximately the size of the laser spot, is 1-2 $\pi \mathrm{mm}$-mrad, competitive with state of the art radio-frequency facilities.

To understand the details of the LWFA, particle-in-cell simulations using the code VORPAL (Nieter \& Cary 2004) (developed at the University of Colorado and Tech-X Corporation) were conducted in parameter regimes relevant to the experiments (Geddes et al. 2004a). In these simulations, it is observed that in the first few hundred microns of propagation of the laser pulse in the channel, the wake amplitudes (and hence the number of trapped particles) are small. As the laser pulse envelope starts distorting through the self-modulation instability, developing features with rise times on the order of or shorter than the plasma period, a plasma wake is excited that is large enough to trap and accelerate particles. Once enough charge is accumulated in the accelerating bucket, the injection process can be terminated due to beam loading, i.e., the field of the accelerated bunch modifies the wakefield and reduces its amplitude to below the trapping threshold. Pump depletion of the laser pulse energy (lost to wake excitation) can also reduce the wake amplitude below the trapping threshold. 


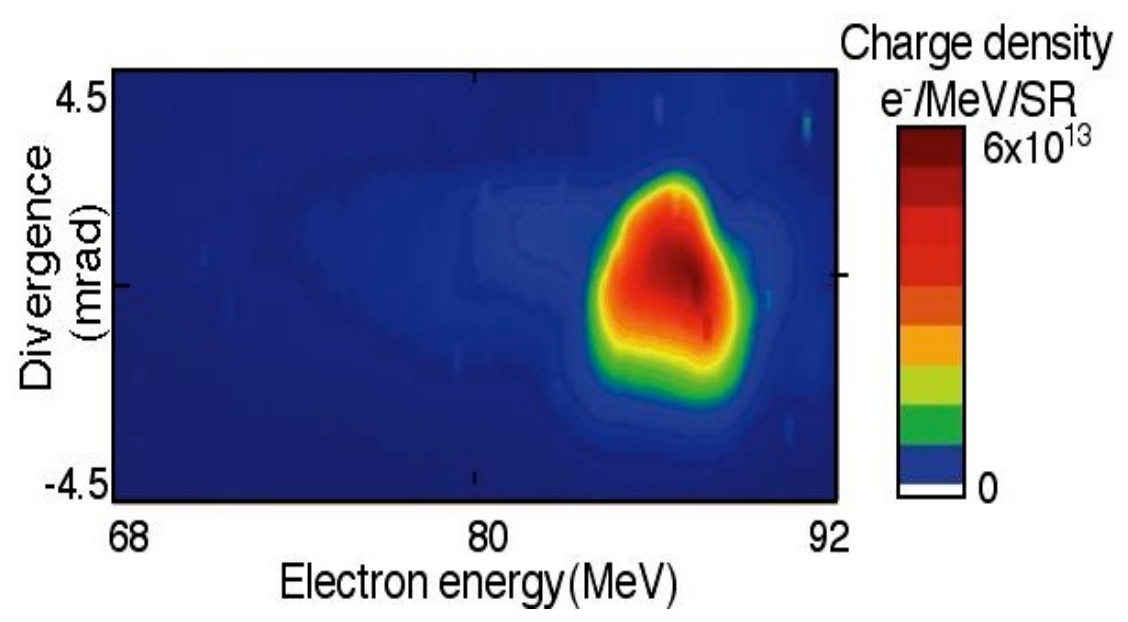

Figure 3. Electron energy spectrum of a bunch produced by the channel guided accelerator. The spectrum was obtained by dispersing the electron beam with the $55^{\circ}$ magnetic spectrometer and recording the beam image on a phosphor screen imaged with a high resolution CCD-camera. The energy range covered in this single shot is from $68-92 \mathrm{MeV}$ and shows the appearance of mono-energetic features, here with $3 \times 10^{9}$ electrons in a bunch with energy spread of $4 \% \mathrm{FWHM}$ at $78 \mathrm{MeV}$. In the vertical (non-dispersive) plane, the divergence was near 3 mrad FWHM for this bunch.

The electron energy spread is minimized if the acceleration process is terminated after a dephasing distance, i.e., when the bunch is at its maximum energy at the top of the separatrix. The momentum bunching that occurs at the top of the bucket results in high quality electron beams. By carefully controlling the accelerator length to match the dephasing distance, high energy electron beams with narrow energy spread can hence be obtained. If the trapped electrons propagate beyond a dephasing distance, the electrons lose energy, which leads to a broad energy distribution.

Reaching the dephasing distance was also put forward by the RAL and LOA groups as the mechanism behind their observation of narrow energy spread bunches (Mangles et al. 2004; Faure et al. 2004).

\section{Summary and future developments}

\section{(a) High intensity laser guiding and mono-energetic electron beams}

During the past few years, two important milestones towards the development of a laser-plasma accelerator have been achieved: laser guiding at high intensity and production of mono-energetic beams. Using preformed plasma channels, laser beams of relativistic intensities (i.e., $a_{0}^{2} \gg 1$ ) have been guided over $>10 Z_{R}$ (Geddes et al. 2004a). Two regimes were found at LBNL. In the first regime, intense laser pulses at the 4 TW power level were guided and transmission levels approaching 50 percent were obtained. No significant amount of accelerated charge was detected. This result shows that it is possible to guide laser pulses over macroscopic distances at relativistic intensities without generating self-trapped electrons or "dark 
current". This is an important result for future development of laser accelerator modules that rely on the use of wakefield excitation.

In the second regime, intense laser pulses were guided and high energy electron beams were observed that had an energy distribution with narrow structure. Using a magnetic spectrometer, isolated intense monoenergetic beams were produced at the $100 \mathrm{MeV}$ energy level with energy spread at the level of a few percent, containing several 100's of picoCoulomb of charge. Transversely, these bunches were found to have a normalized emittance of $1-2 \pi \mathrm{mm}-\mathrm{mrad}$, limited by the resolution of the diagnostic. In this regime, the transmitted laser pulse was severely depleted.

Narrow energy spread electron beams were also observed at LOA and RAL without relying on guiding but instead using $20 \mu \mathrm{m}$ scale laser spot sizes with long, lower density plasmas. Similarly, at LBNL narrow energy spread beams were obtained using $8.5 \mu \mathrm{m}$ laser spots and short, higher density plasmas without guiding or long, lower density plasmas with self-guiding (Geddes et al. 2004a; Geddes et al. 2005). The fundamental reason for the observation of these narrow energy spread beams seems consistent with matching the acceleration distance to the dephasing distance of an electron in the plasma wave bucket. This was confirmed via particlein-cell simulations and via experiments at LBNL in which a single beam was used in gas jets of various lengths and gas densities.

Based on experiment, simulation, and theory, the production of monoenergetic bunches in a laser-plasma accelerator requires the following four steps: Step 1 consists of exciting a wakefield. For a self-modulated LWFA, this typically occurs after the laser has propagated a sufficiently long distance within the plasma, such that the self-modulation instability (i.e., the feedback of the wake on the pulse and the self-consistent evolution of both the wake and the pulse) excites a large amplitude wakefield. Step 2 consists of a method for trapping and the initial injection of the electrons into the wake. For a self-modulated LWFA, this can be the result of selftrapping. Step 3 consists of termination of the self-trapping or injection process. If trapping is not terminated, low energy electrons would continuously be injected into the wake over the entire length of acceleration, resulting in a large energy spread. One mechanism to accomplish this is by beam loading, i.e., the injected electron bunch is of sufficient charge so as to reduce the amplitude of the wake below the self-trapping threshold. Step 4 is acceleration of the electron bunch over a distance equal to the dephasing length. If acceleration occurs over distances longer than the dephasing length, the trapped bunch will continue to circulate around the separatrix, losing energy and increasing its energy spread. Optimum acceleration would occur over a distance equal to the dephasing length, such that the trapped bunch exits the plasma near the top of the separatrix (i.e., the accelerating phase-space bucket), with maximum energy and minimum energy spread.

In the LBNL experiments, matching of the acceleration length and the dephasing length was obtained by using a preformed plasma channel. The length of the channel can be adjusted to control the acceleration length, whereas the channel depth and radius can be adjusted to control the dephasing length (via the wake phase velocity). In the LOA and RAL experiments, matching of the acceleration length to the dephasing length was the result of using a larger laser spot size (i.e., a larger $Z_{R}$ and a longer acceleration length) along with the use of lower plasma densities, which increases the dephasing length. If a large $Z_{R}$ is used in combination with high plasma densities, then the acceleration length would exceed the dephasing 
length, and large energy spreads are expected. This was in fact observed in the LOA and RAL experiments, as well as in the LBNL experiments that relied on selfguiding (i.e., no preformed channel) (Geddes et al. 2004b; 2005). At high densities, a continuous Boltzmann-like distribution was observed. As the density was decreased, narrow energy spread peaks began to emerge in the energy spectrum.

It should be noted that, without detectors capable of covering the entire dispersive area of the spectrometer, narrow energy spread features can easily be missed. Many previous experiments used Si-based diodes for electron detection, with limited energy coverage per channel that could easily have missed narrow energy spread beams. Present experiments all use continuous phosphor screens with CCD imagers.

\section{(b) Quantifying the progress}

To quantify the progress made in laser driven accelerators, figures 4 and 5 show the total beam energy and brightness versus particle energy, respectively, for the beams produced in the three papers published in the 30 September 2004 issue of Nature. Expected performance of $1 \mathrm{GeV}$ and $10 \mathrm{GeV}$ modules are also plotted. The spread in beam energy and brightness is due to the uncertainty in predicting the normalized emittance and total charge of the beam for those two cases. The upper limit in brightness assumes that the normalized emittance measured for the $100 \mathrm{MeV}$-class beams will be conserved when accelerating to 1 or $10 \mathrm{GeV}$, whereas the lower limit assumes that the divergence angle will be conserved. For comparison, the same quantities are plotted for a conventional thermionic gun, a photo-cathode radio-frequency gun (Yakimenko et al. 2002) and the $30 \mathrm{GeV}$ beam at the Stanford Linear Accelerator Center (SLAC). As can be seen in figure 4 beam energy is comparable for the different $100 \mathrm{MeV}$-class linacs, but, for the $>10 \mathrm{GeV}$ laser accelerators, considerable progress must be made in increasing the total beam energy to become competitive. From figure 5 it can be seen that in the shorter term, laser driven accelerators can have an advantage over conventional accelerators due to the high peak brightness which is a direct result of the ultra-short pulse nature of the bunches and the competitively low normalized emittance.

\section{(c) Future challenges}

The immediate challenge ahead is to stabilize the performance of the accelerator. The charge per bunch integrated over all energies is found to be relatively stable, at the few percent level, in the high repetition LBNL experiments. The bunch energy and amount of charge in the narrow energy spread beams, however, fluctuates. This is expected to be remedied with sophisticated control over the electron trapping process as well as the laser and plasma source operation. Since the energy depends linearly on laser intensity [see, e.g., equation (2.2)], control of the pulse length, pulse energy, and spot size is needed at the few percent level. Pulse length control requires spectral and pointing control. In the laser pulse stretcher and compressor, for example, pointing fluctuations translate into pulse duration fluctuation. To minimize pointing fluctuations, great care must be taken during the construction of future systems to precisely control the environment (temperature and vibration). Pointing errors can also translate into pulse energy changes, due to changes in overlap between the gain medium and the amplified pulse. Novel meth- 




Figure 4. Electron beam energy (number of particles times mean particle energy per bunch) vs. particle energy for a conventional RF linacs (thermionic gun and a photo-cathode RF gun), the SLAC FFTB beam, beams reported in the 30 September 2004 Nature issue and expected performance of a $1 \mathrm{GeV}$ and a $10 \mathrm{GeV}$ laser-driven plasma accelerator.

ods for controlling laser pulse energy are being developed in industry for short pulse systems that may meet these requirements. The origin of spot size fluctuations is not entirely understood, but may also be affected by pump laser changes that can affect the divergence, and overall spot size of the amplified beam (as well as beam pointing).

Plasma densities must be controlled at the percent level to ensure that the wake amplitude $\left(\propto n^{1 / 2}\right)$, dephasing length $\left(\propto 1 / n^{3 / 2}\right)$ and energy gain $(\propto 1 / n)$ remain constant. In addition to the development of plasma density diagnostics capable of providing this level of accuracy, efforts are required to engineer pulsed gas jet systems that limit the shot-to-shot variation. When using statically filled chambers, such as in the case of a hydrogen based capillary discharge system (Spence \& Hooker 2001), gas densities should be controllable to a sufficient level. This should result in reproducible plasma densities, provided the electrical discharge is reproducible.

As has been discussed, matching the accelerator length to the dephasing length can produce narrow energy spread beams, even when relying on self-trapping. However, accurate control of the performance of the accelerator will most likely require a method for triggering the trapping process. Various methods for injecting electrons into the wake with one or more additional laser pulses have been proposed (Umstadter et al. 1996b; Esarey et al. 1997; Hemker et al. 1998; Schroeder et al. 


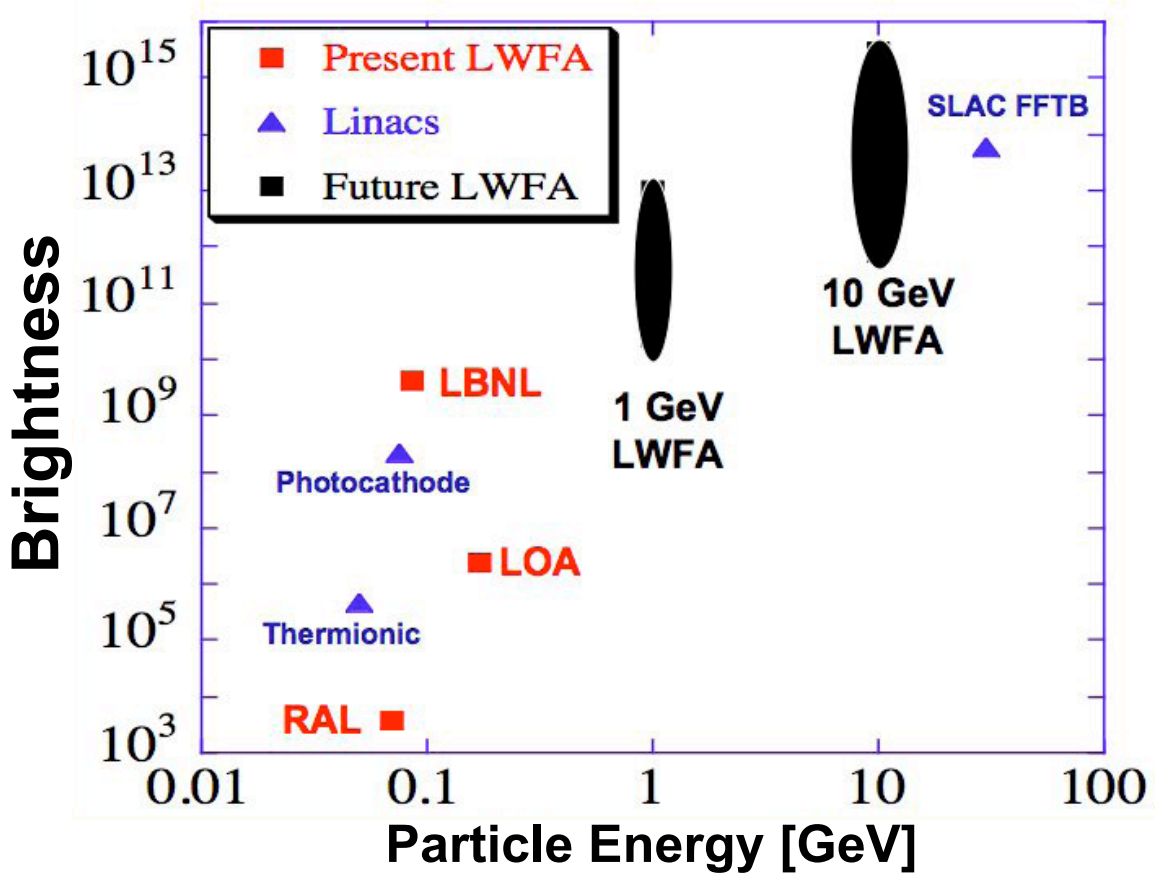

Figure 5. Electron beam brightness vs. particle energy for a conventional thermionic gun, a photo-cathode RF gun, the SLAC FFTB beam, beams reported in the 30 September 2004 Nature issue, and expected performance of a $1 \mathrm{GeV}$ and a $10 \mathrm{GeV}$ laser driven accelerator. The brightness is calculated by multiplying the total number of particles with the mean bunch energy and dividing by the bunch duration, the relative energy spread and the square of the unnormalized emittance (in $\mathrm{mm}^{2}-\mathrm{mrad}^{2}$ ).

1999; Fubiani et al. 2004). Experiments are underway to study laser triggered injection, and are predicted by theory to produce narrow energy spread bunches with greater stability.

Combined with channeling technology, optically triggered injection, we believe, forms the basis of an all-optical $\mathrm{GeV}$ accelerator. As can be seen from the basic scaling laws, reducing the density and lengthening the distance over which the plasma channel extends is essential to reach GeV energies. To achieve multi-cm scale plasma channels, novel methods relying on hydrogen capillary discharges developed by Hooker et al. (Spence \& Hooker 2001) are being tested with the 100 TW LOASIS amplifier. To reduce the amount of energy required from the laser system in schemes that use inverse Bremsstrahlung to heat the plasmas (hydrodynamically formed channels), cluster jets will be tested such as being developed by Milchberg et al. (Taguchi et al. 2004).

The next challenge will be to go beyond GeV energy levels, requiring a demonstration of staging different modules, as well as development of laser systems capable of delivering high peak power, and large amounts of energy, at high repetition rate (i.e., high average power). As a simple example, consider producing a $10 \mathrm{GeV}$ elec- 
tron beam, containing $1 \mathrm{nC}$ of charge $\left(6 \times 10^{9}\right.$ electrons). This represents $10 \mathrm{~J}$ worth of energy and assuming a laser to particle beam efficiency between $1-10 \%$, requires therefore 100-1000 J/pulse of laser energy. It is obvious from this simple calculation that it is essential for both accelerator and laser development to be pursued, if one wants to realize the goal of all-optical linacs. Such an accelerator holds the promise of offering unique beams, having femtosecond duration and containing 100's of pC of charge, with an emittance that equals or surpasses conventional linacs. If the development continues to be successful, it will serve as a compact multi-GeV injector for high energy physics applications, as well as the basis for novel radiation sources including next generation femtosecond light sources.

\section{(d) Is the use of a channel the only way to go?}

Can channeling be avoided by using ever more powerful laser beams? Based on the first three experiments that demonstrated the production of mono-energetic electron beams, it is clear that for fixed laser power unchanneled experiments accelerated less charge and reached lower energy than channeled experiments; increasing the laser spot size decreased its intensity and hence the wake amplitude. To further study this question, consider the high intensity limit, $a_{0}>1$. Recalling that the selffield of a point charge extends out to a radius of order $k_{p}^{-1}=\lambda_{p} / 2 \pi$, the constraint $k_{p} r_{0} \sim O(1)$ and hence $w_{0} \propto \lambda_{p}$ is necessary to ensure efficient coupling between the wakefield and the electron bunch. For the diffraction limited case, the energy gain can be written as $\Delta W_{\text {diff }}[\mathrm{MeV}] \simeq 0.8\left(k_{p} w_{0}\right)(P[\mathrm{GW}])^{1 / 2}$. For this to apply, it is necessary for $P<P_{c}=17\left(\lambda_{p} / \lambda\right)^{2} \mathrm{GW}$ (Esarey et al. 1996), so that the effects of relativistic self-focusing can be neglected, and for $Z_{R}<L_{\text {deph }} \simeq a_{0} \lambda_{p}^{3} /\left(2 \lambda^{2}\right)$, so that dephasing can be neglected. Note that $Z_{R}<L_{\text {deph }}$ can be written as $\left(k_{p} w_{0}\right)^{2}<2 \pi a_{0} \lambda_{p} / \lambda$ or $\left(k_{p} w_{0}\right)^{3}<8.5(P[\mathrm{GW}])^{1 / 2}$. Since $Z_{R}<L_{\text {deph }}$ is easily satisfied and $\Delta W_{\text {diff }} \propto P^{1 / 2}$, the energy gain will be strongly limited without some form of guiding.

Using a density channel for laser pulse guiding the dephasing-limited energy gain can be written as $\Delta W_{\text {deph }}[\mathrm{MeV}] \simeq 0.9\left(k_{p} w_{0}\right)^{-2} P[\mathrm{GW}]$ (for $a_{0}>1$ and $k_{p} w_{0} \sim$ $O(1)$ ), e.g., $P \simeq 100 \mathrm{TW}$ and $k_{p} w_{0} \simeq 4$ give $\Delta W_{\text {deph }} \sim 6 \mathrm{GeV}$.

For sufficiently high powers $P \gg P_{c r}$, it may be possible to guide the laser pulse over multiple $Z_{R}$ without the use of a density channel due to a combination of relativistic self-focusing and ponderomotive self-channeling. This is the case in the so-called blow-out or bubble regime (Pukhov \& Meyer-ter-Vehn 2002). Assuming that the energy gain is limited by dephasing with $a_{0}>1$ and $k_{p} r_{0} \sim 1$ again implies $\Delta W_{\text {deph }}[\mathrm{MeV}] \simeq 0.9\left(k_{p} r_{0}\right)^{-2} P[\mathrm{GW}]$, only now with the additional constraint $P \gg$ $P_{c r}$. Note that if $P=R_{c r} P_{c r}$, where $R_{c r} \sim 10$ (typical value considered in present studies $)$ is a constant, then this implies $a_{0}=5.6 R_{c r}^{1 / 2} /\left(k_{p} w_{0}\right)$. Similar scalings for the energy, i.e., $\Delta W \propto P$, can also be obtained through analytical and numerical studies of the blow-out regime. For example, Gordienko \& Pukhov (2005) obtain $\Delta W_{G P}[\mathrm{MeV}] \simeq 0.1\left(c \tau_{L} / \lambda\right)(P[\mathrm{GW}])^{1 / 2}$. But if $c \tau_{L}=R_{p} \lambda_{p}$, where $R_{p} \sim 1$ is a constant, and if $P=R_{c r} P_{c r}$, then $\Delta W_{G P}[\mathrm{MeV}] \simeq 0.03 R_{p} R_{c r}^{-1 / 2} P[\mathrm{GW}]$. Mori (2005, personal communication) finds $\Delta W_{M}[\mathrm{MeV}] \simeq 0.5\left(\lambda_{p} / \lambda\right)^{4 / 3}(P[\mathrm{GW}])^{1 / 3}$, which can be rewritten as $\Delta W_{M}[\mathrm{MeV}] \simeq 0.02 R_{c r}^{-2 / 3} P[\mathrm{GW}]$ assuming $P=R_{c r} P_{c r}$.

For the specific case where $k_{p} w_{0} \simeq 4$ and $R_{c r} \sim 10$, substitution of these values in the expressions given above reveals that the particle energy gain with guiding 
is more than an order of magnitude larger than in a single unguided stage, for the same initial laser power.

Although $\Delta W \sim P$ when limited by dephasing for both a channel-guided LWFA or a self-guided LWFA with $P \gg P_{c r}$, there may be additional advantages to using a channel over relying on self-guiding. One obvious difference is that the additional constraint $P \gg P_{c r}$ need not be satisfied when using a channel. This implies that the channel-guided LWFA may be operated at lower intensities (lower $a_{0}$ ), which may be a more stable regime. The channel may also provide some resistance to instabilities, such as the laser-hose instability. Without a channel, the laser pulse will be subject to some amount of diffractive erosion, since the head of the pulse will not be self-guided, which can limit the propagation distance. For example, if the pulse is self-guided for a distance of $L=R_{R} Z_{R}$, where $R_{R} \gg 1$ is the number of $Z_{R}$, then erosion will limit the energy gain instead of dephasing. This will be the case when $R_{R} Z_{R}<L_{\text {deph }}$, or, alternatively $R_{R}\left(k_{p} r_{0}\right)^{3}<8.5(P[\mathrm{GW}])^{1 / 2}$. Lastly, it is hoped that by operating a channel-guided LWFA in the "dark-current-free" mode (no self-trapping), a high quality electron bunch can be obtained by injecting a low energy spread, low emittance bunch into the LWFA. If the LWFA is to be operated in the self-guided mode (i.e., the blow-out or bubble regime), it may not be possible to operate in this regime without self-trapping, which may limit the achievable energy spread and emittance of the accelerated bunch.

The authors acknowledge Catalin Filip, Gwenael Fubiani, Estelle Michel, Pierre Michel, Bob Nagler, Kei Nakamura, Brad Shadwick, Jeroen van Tilborg, Simon Hooker, Tony Gonsalves, John Cary, David Bruhwiler, Dimitre Dimitrov, and Chet Nieter for all their help. This work was supported by the Department of Energy, Office of Science, High Energy Physics under contract DE-AC03-76SF0098.

\section{References}

Alexeev, I., Antonsen Jr, T. M., Kim, K. Y. \& Milchberg, H. M. 2003 Self-focusing of intense laser pulses in a clustered gas. Phys. Rev. Lett. 90, 103402.

Bulanov, S. V., Inovenkov, I. N., Kirsanov, V. I., Naumova, N. M. \& Sakharov, A. S. 1992 Nonlinear depletion of ultrashort and relativistically strong laser pulses in an underdense plasma. Phys. Fluids B 4, 1935-1942.

Catravas, P., Esarey, E. \& Leemans, W. P. 2001 Femtosecond x-rays from Thomson scattering using laser wakefield accelerators. Meas. Sci. Technol. 12, 1828-1834.

Cros, B., Courtois, C., Matthieussent, G., Bernardo, A., Di Batani, D., Andreev, N. \& Kuznetsov, S. 2002 Eigenmodes for capillary tubes with dielectric walls and ultraintense laser pulse guiding. Phys. Rev. E65, 026405.

Downer, M. C., Chiu, C., Fomyts'kyi, M., Gaul, E. W., Grigsby, F., Matlis, N. H., Shim, B., Smith, P. J. \& Zgadzaj, R. 2003 Plasma Channels and laser pulse tailoring for GeV laser-plasma accelerators. In Advanced Accelerator Concepts. Tenth Workshop (eds. C. E. Clayton \& P. Muggli). AIP Conf. Proc., no. 647, pp. 654-663.

Durfee III, C. G. \& Milchberg, H. M. 1993 Light pipe for high intensity laser pulses. Phys. Rev. Lett. 71, 2409-2411.

Esarey, E., Sprangle, P., Krall, J. \& Ting, A. 1996 Overview of plasma-based accelerator concepts. IEEE Trans. Plasma Sci. 24, 252-288.

Esarey, E., Hubbard, R. F., Leemans, W. P., Ting, A. \& Sprangle, P. 1997 Electron injection into plasma wake fields by colliding laser pulses. Phys. Rev. Lett. 79, 26822685 . 
Esarey, E., Shadwick, B. A., Catravas, P. \& Leemans, W. P. 2002 Synchrotron radiation from electron beams in plasma-focusing channel. 65, 056505.

Esarey, E., Shadwick, B. A., Schroeder, C. B. \& Leemans, W. P. 2004 Nonlinear pump depletion and electron dephasing in laser wakefield accelerators. In Advanced Accelerator Concepts. Eleventh Workshop (ed. V. Yakimenko). AIP Conf. Proc., no. 737, pp. 578584.

Faure, J., Glinec, Y., Pukhov, A., Kiselev, S., Gordienko, S., Lefebvre, E., Rousseau, J.P., Burgy, F. \& Malka, V. 2004 A laser plasma accelerator producing monoenergetic electron beams. Nature 431, 541-544.

Fubiani, G., Esarey, E, Schroeder, C. B. \& Leemans, W. P. 2004 Beat wave injection of electrons into plasma waves using two interfering laser pulses. Phys. Rev. E 70, 016402.

Geddes, C. G. R., Tóth, Cs., van Tilborg, J., Esarey, E., Schroeder, C. B., Bruhwiler, D., Nieter, C., Cary, J. \& Leemans, W. P. 2004a High quality electron beams from a plasma channel guided laser wakefield accelerator. Nature 431, 538-541.

Geddes, C. G. R., Tóth, Cs., van Tilborg, J., Esarey, E., Schroeder, C. B., Bruhwiler, D., Nieter, C., Cary, J. \& Leemans, W. P. 2004b Laser guiding at relativistic intensities and wakefield particle acceleration in plasma channels. In Advanced Accelerator Concepts. Eleventh Workshop (ed. V. Yakimenko). AIP Conf. Proc., no. 737, pp. 521-527.

Geddes, C. G. R., Tóth, Cs., van Tilborg, J., Esarey, E., Schroeder, C. B., Bruhwiler, D., Nieter, C., Cary, J. \& Leemans, W. P. 2005 Production of high quality electron bunches by dephasing and beam loading in channeled and unchanneled laser plasma accelerators. Phys. Plasmas 12 , 056709.

Gordienko, S. \& Pukhov, A. 2005 Scalings for ultrarelativistic laser plasmas and quasimonoenergetic electrons. Phys. Plasmas 12043109.

Hemker, R. G., Tzeng, K.-C., Mori, W. B., Clayton, C. E. \& Katsouleas, T. 1998 Computer simulations of cathodeless, high-brightness electron-beam production by multiple laser beams in plasmas. Phys. Rev. E 57, 5920-5928.

Horton, W. \& Tajima, T. 1986 Pump depletion in the plasma-beat-wave accelerator. Phys. Rev. A 34, 4110-4119.

Ledingham, K. W. D., McKenna, P. \& Singhal, R. P. 2003 Applications for nuclear phenomena generated by ultra-intense lasers. Science 300, 1107.

Leemans, W. P., Clayton, C. E., Mori, W. B., Marsh, K. A., Kaw, P. K., Dyson, A., Joshi, C. \& Wallace, J. M. 1992 Experiments and simulations of tunnel-ionized plasmas. Phys. Rev. A 46, 1091-1105.

Leemans, W. P., Siders, C. W., Esarey, E., Andreev, N. E., Shvets, G. \& Mori, W. B. 1996 Plasma guiding and wakefield generation for second-generation experiments. IEEE Trans. Plasma Sci. 24, 331-342.

Leemans, W. P., Chattopadhyay, C., Esarey, E., Zholents, A., Zolotorev, M., Chin, A., Schoenlein, R. \& Shank, C. 2000 Femtosecond x-ray generation through relativistic electon beam-laser interaction. Comptes Rendus de l'Academie des Sciences Serie IV Physique Astrophysique 1, 279-296.

Leemans, W. P., Rodgers, D., Catravas, P., Geddes, C. G. R., Fubiani, G., Esarey, E., Shadwick, B. A., Donahue, R. \& Smith, A. 2001 Gamma-neutron activation experiments using laser wakefield accelerators. Phys. Plasmas 8 2510-2516.

Leemans, W. P., et al. 2002 Electron-yield enhancement in a laser-wakefield accelerator driven by asymmetric laser pulses. Phys. Rev. Lett. 89174802.

Lopes, N. C., et al. 2003 Plasma channels produced by a laser-triggered high-voltage discharge. Phys. Rev E 68, 035402.

Malka, V., et al. 2002 Electron acceleration by a wake field forced by an intense ultrashort laser pulse. Science 298, 1596-1600. 
Mangles, S., et al. 2004 Monoenergetic beams of relativistic electrons from intense laserplasma interactions. Nature 431, 535-538.

Modena, A., et al. 1995 Electron acceleration from the breaking of relativistic plasma waves. Nature 377, 606-608.

Nieter, C. \& Cary, J. 2004 VORPAL: a versatile plasma simulation code. J. Comp. Phys. 196, 448.

Pukhov, A. \& Meyer-ter-Vehn, J. 2002 Laser wake field acceleration: the highly non-linear broken-wave regime. Appl. Phys. B 74 355-361.

Rankin, R., Capjack, C. E., Burnett, N. H. \& Corkum, P. B. 1991 Refraction effects associated with multiphoton ionization and ultrashort-pulse laser propagation in plasma waveguides. Opt. Lett. 16, 835-837.

Rosenzweig, J. B., Breizman, B., Katsouleas, T. \& Su, J. J. 1991 Acceleration and focusing of electrons in two-dimensional nonlinear plasma wake fields. Phys. Rev. E 44, R6189R6192.

Rousse, A., et al. 2004 Production of a keV x-ray beam from synchrotron radiation in relativistic laser-plasma interaction. Phys. Rev. Lett. 93, 135005.

Santala, M. I. K., et al. 2001 Observation of a hot high-burrent electron beam from a self-modulated laser wakefield accelerator. Phys. Rev. Lett. 86, 1227-1230.

Schroeder, C. B., Lee, P. B., Wurtele, J. S., Esarey, E. \& Leemans, W. P. 1999 Generation of ultrashort electron bunches by colliding laser pulses. Phys. Rev. E 59 6037-6047.

Schroeder, C. B., Esarey, E., Geddes, C. G. R., Tóth, Cs., Shadwick, B., van Tilborg, J., Faure, J. \& Leemans, W. P. 2003 Frequency chirp and pulse shape effects in selfmodulated laser wakefield accelerators. Phys. Plasmas 10, 2039-2046.

Schroeder, C. B., Esarey, E., van Tilborg, J. \& Leemans, W. P. 2004 Theory of coherent transition radiation generated at a plasma-vacuum interface. Phys. Rev. E69, 016501.

Spence, D. J. \& Hooker, S. M. 2001 Investigation of a hydrogen plasma waveguide. Phys. Rev. E 63, 015401.

Sprangle, P., Esarey, E., Krall, J. \& Joyce, G. 1992 Propagation and guiding of intense laser pulses in plasmas. Phys. Rev. Lett. 69, 2200-2203.

Sprangle, P., et al. 2001 Wakefield generation and $\mathrm{GeV}$ acceleration in tapered plasma channels. Phys. Rev. E 63, 056405.

Strickland, D. and Mourou, G. Opt. Comm. 1985 56, 219.

Taguchi, T., Antonsen Jr, T. M. \& Milchberg, H. M. 2004 Resonant Heating of a cluster plasma by intense laser light. Phys. Rev. Lett. 92, 205003.

Tajima, T. \& Dawson, J. M. 1979 Laser electron accelerator. Phys. Rev. Lett. 43, 267-270.

Tochitsky, S. Y., et al. 2004 Enhanced acceleration of injected electrons in a laser-beatwave-induced plasma channel. Phys. Rev. Lett. 92, 095004.

Umstadter, D., Chen, S.-Y., Maksimchuk, A., Mourou, G. \& Wagner, R. $1996 a$ Nonlinear optics in relativistic plasmas and laser wake field acceleration of electrons. Science $\mathbf{2 7 3}$ $472-475$.

Umstadter, D., Kim, J. K. \& Dodd, E. $1996 b$ Laser injection of ultrashort electron pulses into wakefield plasma waves. Phys. Rev. Lett. 76, 2073-2076.

Volfbeyn, P., Esarey, E. \& Leemans, W. P. 1999 Guiding of laser pulses in plasma channels created by the ignitor-heater technique Phys. Plasmas 6, 2269-2277.

Xiao, Y.-F., Chu, H.-H., Tsai, H.-E., Lee, C.-H., Lin, J.-Y., Wang, J. \& Chen, S.-Y. 2004 Efficient generation of extended plasma waveguides with the axicon ignitor-heater scheme Phys. Plasmas 11, L21-L24.

Yakimenko, V., Babzien, M., Ben-Zvi, I., Malone, R. \& Wang, X.-J. 2002 Submicron emittance and ultra small beam size measurements at ATF Nucl. Inst. and Methods A 483, $277-281$. 
Zhidkov, A., Koga, J., Esirkepov, T., Hosokai, T., Uesaka, M. \& Tajima, T. 2004 Opticalfield-ionization effects on the propagation of an ultraintense laser pulse in high-Z gas jets. Phys. Rev. E 69, 066408. 